\title{
Comparison of prostate contours between conventional stepping transverse imaging and Twister-based sagittal imaging in permanent interstitial prostate brachytherapy
}

\author{
Shogo Kawakami, MD!, Hiromichi Ishiyama, MD!, Takefumi Satoh, MD², Hideyasu Tsumura, MD², Akane Sekiguchi, MD!, \\ Kouji Takenaka, MD', Ken-ichi Tabata, MD², Masatsugu Iwamura, MD², Kazushige Hayakawa, MD' \\ 'Department of Radiology and Radiation Oncology, ${ }^{2}$ Department of Urology, Kitasato University School of Medicine, Kitasato, Sagamihara, \\ Japan
}

\begin{abstract}
Purpose: To compare prostate contours on conventional stepping transverse image acquisitions with those on twister-based sagittal image acquisitions.

Material and methods: Twenty prostate cancer patients who were planned to have permanent interstitial prostate brachytherapy were prospectively accrued. A transrectal ultrasonography probe was inserted, with the patient in lithotomy position. Transverse images were obtained with stepping movement of the transverse transducer. In the same patient, sagittal images were also obtained through rotation of the sagittal transducer using the "Twister" mode. The differences of prostate size among the two types of image acquisitions were compared. The relationships among the difference of the two types of image acquisitions, dose-volume histogram (DVH) parameters on the post-implant computed tomography (CT) analysis, as well as other factors were analyzed.

Results: The sagittal image acquisitions showed a larger prostate size compared to the transverse image acquisitions especially in the anterior-posterior $(\mathrm{AP})$ direction $(p<0.05)$. Interestingly, relative size of prostate apex in AP direction in sagittal image acquisitions compared to that in transverse image acquisitions was correlated to DVH parameters such as $\mathrm{D}_{90}(R=0.518, p=0.019)$, and $\mathrm{V}_{100}(R=0.598, p=0.005)$.

Conclusions: There were small but significant differences in the prostate contours between the transverse and the sagittal planning image acquisitions. Furthermore, our study suggested that the differences between the two types of image acquisitions might correlated to dosimetric results on CT analysis.

J Contemp Brachytherapy 2017; 9, 4: 316-322 DOI: https://doi.org/10.5114/jcb.2017.69807
\end{abstract}

Key words: brachytherapy, low-dose-rate, prostate cancer, transrectal ultrasound.

\section{Purpose}

Transrectal ultrasound (TRUS) has been used as a standard imaging modality for permanent interstitial prostate brachytherapy (PIPB), not only for pre-implant treatment planning but also for real-time monitoring during implantation $[1,2]$. Because TRUS for PIPB is equipped with a biplane transducer, two types of images, transverse and sagittal, are available in conventional PIPB. Transverse images are acquired through the stepping movement of the transverse transducer, which is set at the tip of the TRUS probe, while sagittal images are acquired through the rotation of the TRUS probe within the rectal cavity (without a stepping movement). Although different imaging techniques can produce differences in the acquired images, to the best of our knowledge, there are few reports regarding the difference between these two types of image acquisitions in PIPB.

Our treatment planning software $\left(\right.$ VariSeed $^{\circledR}$, version 8.0.2, Varian Medical Systems, Palo Alto, CA, USA) has an optional image acquisition program called the "Twister". In this program, sagittal images are available for pre-implant treatment planning in addition to conventional transverse images. Therefore, two types of image acquisitions are available for treatment planning in each patient when the optional Twister program is installed. The purpose of this study was to compare prostate contours in the conventional stepping transverse image acquisitions with those in the Twister-based sagittal image acquisitions. In

\footnotetext{
Address for correspondence: Hiromichi Ishiyama, MD, Department of Radiology and Radiation Oncology, Received: 30.07 .2016 Kitasato University School of Medicine, 1-15-1 Kitasato, Sagamihara, Japan, phone: +81 778-8453, 
addition, the relationships among the difference of the two types of image acquisitions, the dose-volume-histogram (DVH) parameters on the post-implant CT analysis, as well as other factors were analyzed.

\section{Material and methods}

\section{Patients}

The Institutional Review Board approved this prospective study (B14-60). Written informed consent was obtained from all 20 patients who participated in this study. Eligible participants were adults > 20-years-old with localized prostate cancer, clinical stage T1c-T2c, with prostate-specific antigen (PSA) level $<20 \mathrm{ng} / \mathrm{ml}$, and Gleason score $\leq 8$. Exclusion criteria included any contraindications for anesthesia or need for additional external radiotherapy, and refusal to participate. Seven patients had neoadjuvant hormonal therapy. Median duration of hormonal therapy was 5.5 months (range, 4-35 months). The patients' characteristics are shown in Table 1.

\section{Image acquisition}

A TRUS probe (HI VISION Preirus, Hitachi Aloka Medical, Ltd., Tokyo, Japan) was inserted, with the patient in the lithotomy position. Transverse images were acquired through the stepping movement of the transverse transducer set at the tip of the TRUS probe with $1 \mathrm{~mm}$ spacing. In addition, sagittal images of the same prostate were acquired through rotation of the TRUS probe using the Twister mode. These two types of images from each patient were then imported into the planning software and were reconstructed into 3-dimensional volume data. All of these image acquisitions were done before needle insertion. The resolution of TRUS images along with $X, Y, Z$ axis were $0.011 \mathrm{~cm}, 0.011 \mathrm{~cm}$, and $0.100 \mathrm{~cm}$, respectively, for both types of image acquisitions. Quality assurance and quality control of our ultrasound system was performed according to Japanese guideline for seed implantation [3] that was made referring to reports form of American Association of Physicists in Medicine (AAPM) task group.

\section{Prostate size measurements}

Prostates were independently contoured by 2 radiation oncologists and 1 urologist (S.K., H.I., and H.T.) on each slice of transverse image or each transverse plane of the reconstructed 3 -dimensional volume data $(1 \mathrm{~mm}$ slice thickness). Urethra, rectum, and seminal vesicles were not contoured. The investigators could refer to sagittal and coronal plane when they contoured the prostate on transverse plane.

The contoured prostate size was measured in the anterior-posterior (AP) direction at 1) the apex, 2) the midgland, and 3) the base; in the left-right (LR) direction at 4) the apex, 5) the mid-gland, and 6) the base; and in 7) the superior-inferior (SI) direction. In addition, the distances from the probe surface to the anterior prostate edge were measured at 8) the apex, 9) the mid-gland, and 10) the base (Figure 1). The base and the apex were defined as the first and last contoured slice on the image set, and the mid-gland was defined as the slice at midpoint between the two. The volume of contoured prostate were automatically calculated by the software. These measurements were compared between the transverse and the sagittal image acquisitions. The mean values of 3 investigators' measurements were used as the sizes of each prostate. Inter-observer variation was defined as each difference from the mean value of 3 investigators.

\section{Treatment}

An interactive planning technique was used for all patients [4]. After peripheral needle insertion, a transverse TRUS image was acquired again. If needed, the prostate contour was modified based on the second TRUS image because of swelling and deformation of the prostate due to needle insertion. After implantation of radioactive sources with peripheral needles, the interior needles were inserted and the remaining radioactive sources were implanted. If required, the dosimetry was modified based on real-time dose calculation during the procedure.

All treatments were planned only on transverse images using the planning software. The prescribed dose to the prostate was $145 \mathrm{~Gy}$, with a 3- to 5-mm margin. Two types of ${ }^{125} \mathrm{I}$ source were used: either OncoSeed ${ }^{\circledR}$ model 6711 (GE Healthcare [Medi-Physics], Inc, Arlington Heights, IL, USA), or BrachySource ${ }^{\circledR}$ model STM125I (CR BARD, Murray Hill, NJ, USA). Source activities were 11.0 MBq or 13.1 MBq. Both free sources and intraopera-

Table 1. Patients' characteristics

\begin{tabular}{|c|c|c|}
\hline Age (y) & 69 & $(7.2)$ \\
\hline \multicolumn{3}{|l|}{ T stage } \\
\hline $1 c$ & 7 & \\
\hline $2 a$ & 7 & \\
\hline $2 b$ & 3 & \\
\hline $2 c$ & 3 & \\
\hline iPSA (ng/ml) & 6.73 & $(1.75)$ \\
\hline \multicolumn{3}{|l|}{ Gleason score } \\
\hline $3+3$ & 9 & \\
\hline $3+4$ & 4 & \\
\hline $4+3$ & 5 & \\
\hline $4+4$ & 2 & \\
\hline \multicolumn{3}{|c|}{ Hormonal therapy } \\
\hline Yes & 7 & \\
\hline No & 13 & \\
\hline Height $(\mathrm{cm})$ & 165.9 & $(5.8)$ \\
\hline Weight (kg) & 63.4 & $(8.4)$ \\
\hline BMI & 23.0 & $(3.0)$ \\
\hline
\end{tabular}

Values are given as means (standard deviation) or numbers

iPSA - initial prostate-specific antigen, BMI-body mass index 

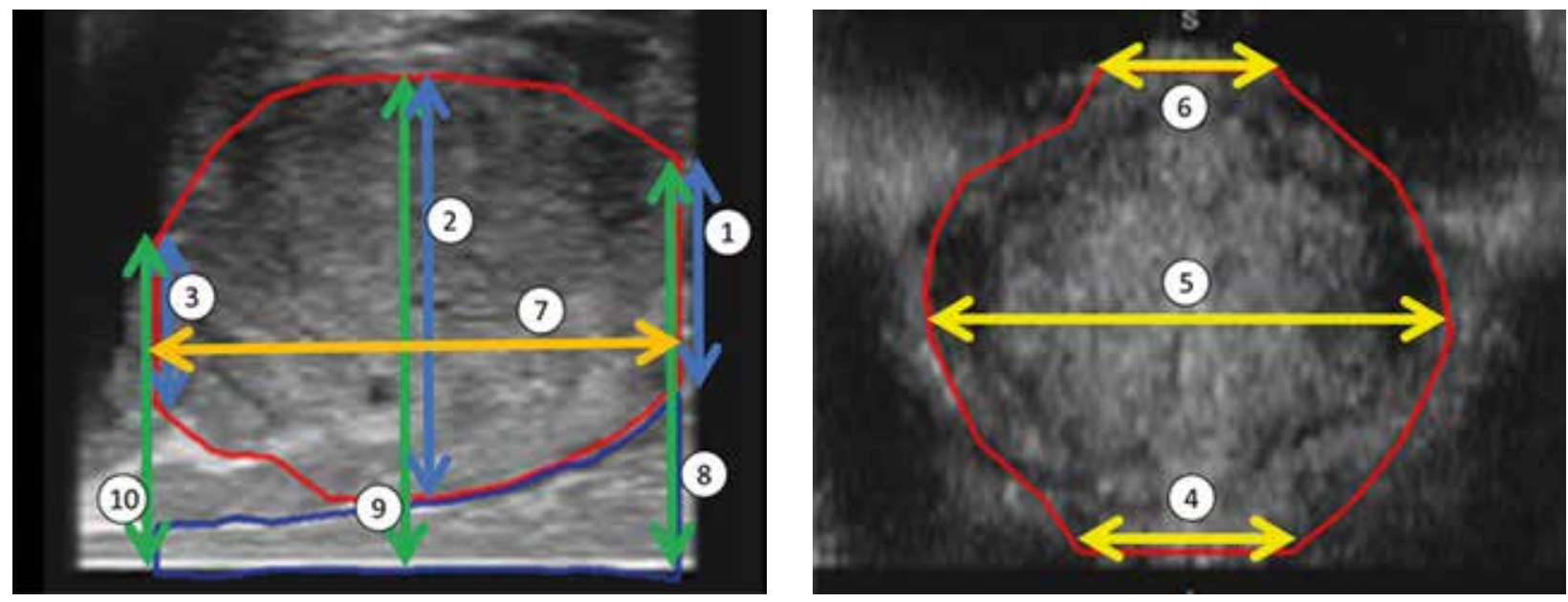

Fig. 1. Prostate size measurement. The blue double-headed arrow $=$ prostate size in the anterior-posterior direction at the 1) apex, 2) mid-gland, and 3) base. The yellow double-headed arrow = prostate size in the left-right direction at the 4) apex, 5) mid-gland, and 6) base. The orange double-headed arrow = prostate size in the 7) superior-inferior direction. The green double-headed arrow $=$ distances from the probe surface to the anterior prostate edge in the 8) apex, 9) mid-gland, and 10) base.

The numbers correspond to those shown in Table 2 and 3

tively built custom-linked (IBCL) sources were used for patients in this study. The free sources were placed one by one transperineally with needles attached to a Mick applicator (Eckert \& Ziegler BEBIG, Berlin, Germany). The IBCL sources were connected to each other using the QUICKLINK system (CR BARD, Murray Hill, NJ, USA) and inserted through a relay system [5]. It has been reported that there is no dosimetric difference between free sources and IBCL sources [6].

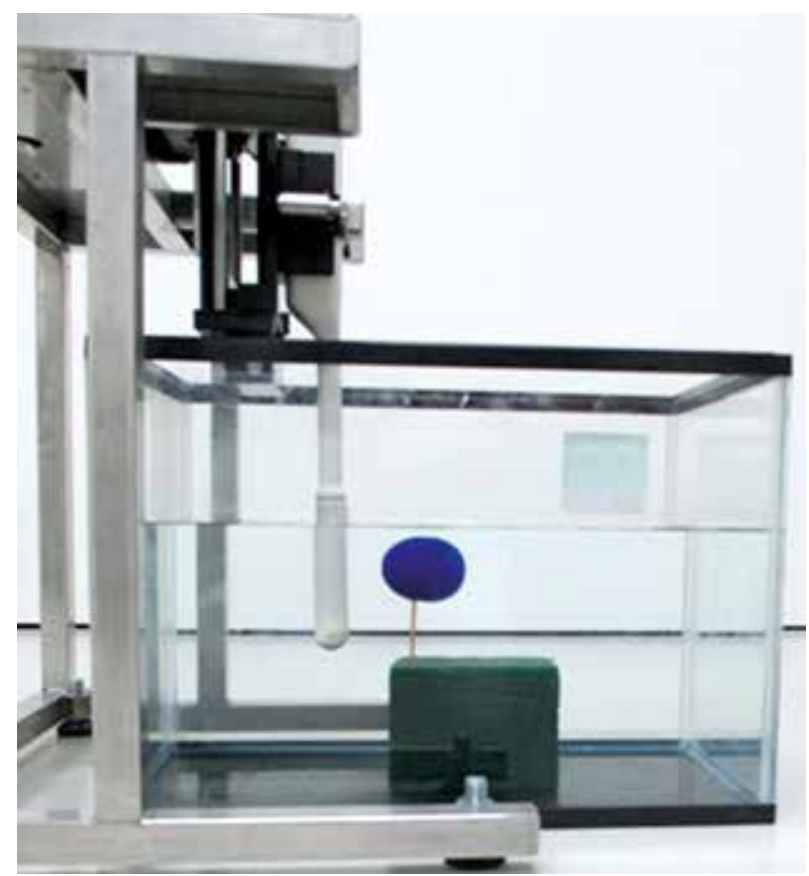

Fig. 2. Set-up for the phantom study. The in-house sphereshaped phantom (blue) was set in a tank of water. The phantom was fixed under the water using weight (green block). Therefore, the phantom could be scanned by transrectal ultrasound (TRUS) without probe pressure

\section{DVH analysis}

Post-implant computed tomography (CT) analysis was completed for all patients based on conventional CT images with $1.25 \mathrm{~mm}$ slice thickness acquired 1 day and 1 month after implantation. In this study, all structures except the urethra were contoured based on the 1 month $\mathrm{CT}$. On the 1 day CT, the outer rim of the urethral catheter was contoured as the urethra. The rectal wall, including the sphincter muscle, was fully contoured on the CT images. The urethra and rectum were contoured in the same slices as the prostate contour. The DVH parameters collected from the CT analysis included the dose to $90 \%$ of the prostate volume $\left(\mathrm{pD}_{90}\right)$; the prostate volume receiving at least $100 \%$ of the dose $\left(\mathrm{pV}_{100}\right)$; the prostate volume receiving at least $150 \%$ of the dose $\left(\mathrm{pV}_{150}\right)$; the urethral volume receiving at least $150 \%$ of the dose $\left(\mathrm{uV}_{150}\right)$; the dose to $30 \%$ of the urethral volume $\left(\mathrm{uD}_{30}\right)$; the dose to $5 \%$ of the urethral volume $\left(\mathrm{uD}_{5}\right)$; the rectal volume receiving at least $100 \%$ of the dose $\left(\mathrm{rV}_{100}\right)$; and the rectal volume receiving at least $150 \%$ of the dose $\left(\mathrm{rV}_{150}\right)$.

\section{A phantom study}

To deny the possibility of image acquisitions themselves causing some differences of prostate size, a phantom study was completed. The in-house oval sphere shaped phantom was set in a tank of water (Figure 2), and was scanned by TRUS using the same methodology as in the above-mentioned human study. CT scan with $1.25 \mathrm{~mm}$ slice thickness was also done for the phantom.

\section{Statistical analysis}

Statistical analyses were performed using R software, version 3.2.0. (R Foundation, Vienna, Austria). The paired $t$-test was used for comparison of the prostate size between the two types of image acquisitions. Pearson's correlation coefficients were calculated for the DVH parameters, 
A

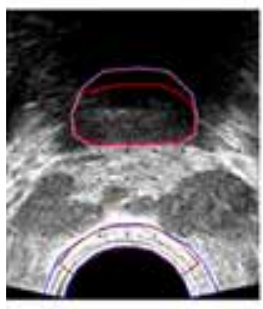

F

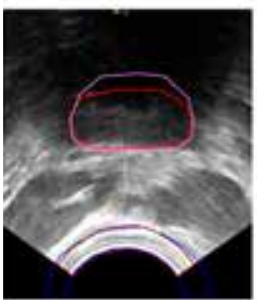

B

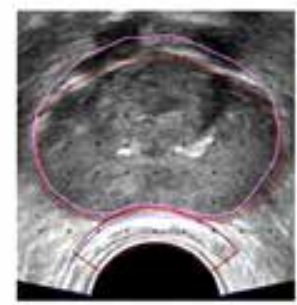

G

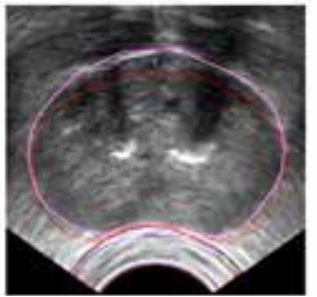

C

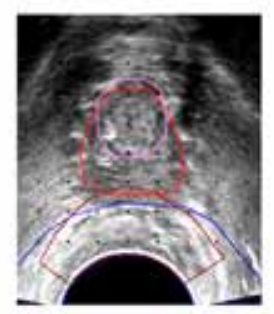

H

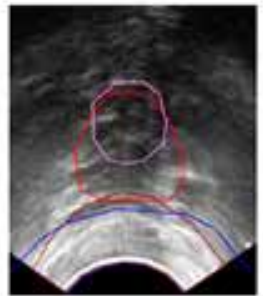

D

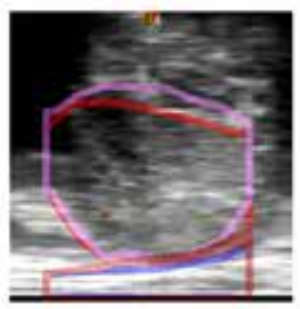

I

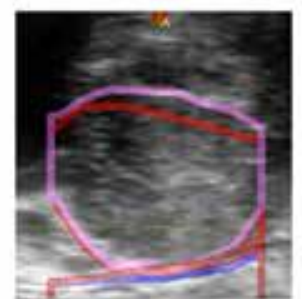

E
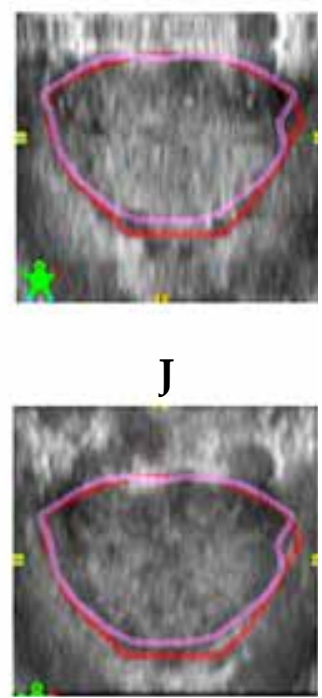

Fig. 3. A representative case showing the difference between the transverse and sagittal image acquisitions. The contours were overlaid based on the transverse (upper row) and sagittal image acquisitions (lower row) at the base (A, F), mid-gland $(B, G)$, apex $(C, H)$. Sagittal plane (D, I) and coronal plane $(E, J)$ of reconstructed 3D volume data were also shown. There was an obvious difference between the transverse-based (red line) and sagittal-based (purple line) contours. In addition, there was an obvious difference in the rectal wall contours (blue for transverse, brown for sagittal)

the differences of the two types of image acquisitions, and the patients' characteristics including body mass index (BMI), age, and with or without hormonal therapy.

\section{Results}

Figure 3 shows a representative case with contours overlaid based on the transverse and sagittal image acquisitions. There was an apparent difference between the transverse-based and the sagittal-based contours. Table 2 shows the comparison of prostate size between the two types of image acquisitions. In the sagittal image acquisitions, the prostate tended to have a longer size in AP and SI direction compared to that in transverse image acquisitions. When the distance from the probe surface to the anterior prostate edge was compared between the two types of image acquisitions, it tended to be longer in the sagittal image acquisitions compared to that in the transverse image acquisitions. In addition, the prostate volume on the sagittal image acquisitions was significantly larger than that on the transverse image acquisitions. Mean inter-observer variation was $0.17 \mathrm{~cm}(\mathrm{SD}=0.10 \mathrm{~cm})$. Figure 4 shows the relationships among the DVH parameters on the post-implant $\mathrm{CT}$ analysis and the difference of the two types of image acquisitions. Interestingly, relative size of prostate apex in AP direction (No. 1 on Figure 1) in sagittal image acquisitions compared to transverse image acquisitions was related to $\mathrm{pD}_{90}$ and $\mathrm{pV}_{100}$ in post-implanted $\mathrm{CT}$ analysis. There was no significant relationships among other DVH parameters and patient's characteristics. Table 3 and Figure 5 show the result of a phantom study. There was no apparent difference in the phantom size among the two types of image acquisitions and CT images. Although about $2 \mathrm{~mm}$ differences in the LR and SI directions were seen between ultrasonography (US) and
CT images, it was probably caused by low visibility of the phantom edge in the CT images (Figure 5).

\section{Discussion}

Our phantom study confirmed that the difference of image acquisitions caused no difference of phantom size. Therefore, the differences in the prostate contours between the two types of image acquisitions would be

Table 2. Comparison of prostate size between transverse and sagittal image acquisitions

\begin{tabular}{|c|c|c|c|}
\hline Directions & Transverse $(\mathrm{cm})$ & Sagittal (cm) & $p$ value \\
\hline 1. AP apex & $1.57 \pm 0.22$ & $1.66 \pm 0.25$ & 0.0346 \\
\hline 2. AP mid-gland & $2.80 \pm 0.33$ & $2.94 \pm 0.37$ & 0.0000 \\
\hline 3. AP base & $1.52 \pm 0.20$ & $1.60 \pm 0.26$ & 0.1819 \\
\hline 4. LR apex & $1.86 \pm 0.30$ & $1.90 \pm 0.32$ & 0.4970 \\
\hline 5. LR mid-gland & $4.50 \pm 0.42$ & $4.45 \pm 0.45$ & 0.3619 \\
\hline 6. LR base & $2.41 \pm 0.30$ & $2.35 \pm 0.34$ & 0.3565 \\
\hline 7. SI & $3.44 \pm 0.35$ & $3.62 \pm 0.38$ & 0.0010 \\
\hline 8. AP edge apex & $2.77 \pm 0.24$ & $3.06 \pm 0.25$ & 0.0000 \\
\hline $\begin{array}{l}\text { 9. AP edge } \\
\text { mid-gland }\end{array}$ & $3.34 \pm 0.34$ & $3.60 \pm 0.33$ & 0.0000 \\
\hline $\begin{array}{l}\text { 10. AP edge } \\
\text { base }\end{array}$ & $2.79 \pm 0.26$ & $2.98 \pm 0.41$ & 0.0068 \\
\hline Volume (ml) & $26.58 \pm 7.82$ & $28.86 \pm 8.19$ & 0.0001 \\
\hline
\end{tabular}


A

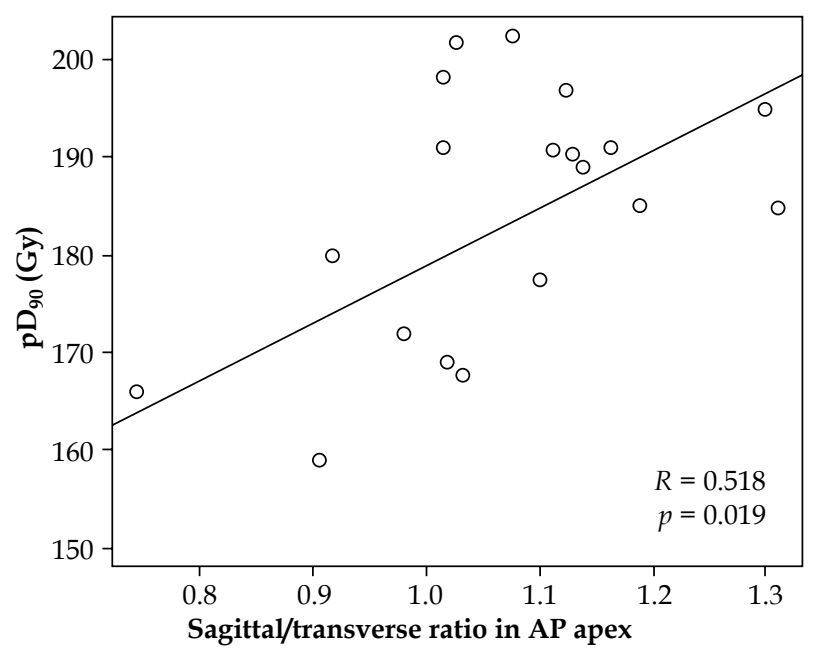

B

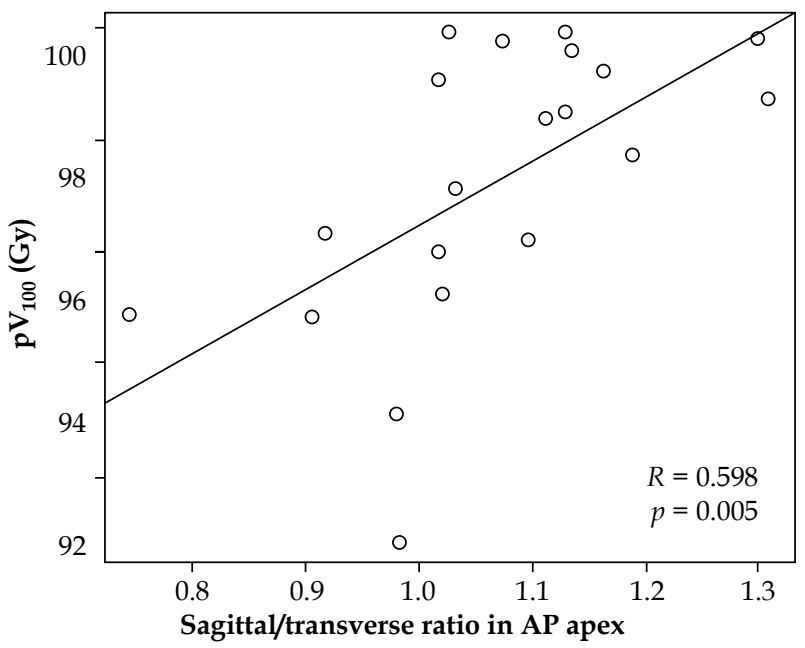

Fig. 4. The relationships in the differences of the 2 types of image acquisitions and the dose-volume histogram parameters on the post-implant computed tomography analysis. The sagittal/transverse ratio in prostate apex in the anterior-posterior direction was related to $\mathrm{pD}_{90}$ and $\mathrm{pV}_{100}$

Table 3. Comparison of the phantom size on the two types of image acquisitions and computed tomography

\begin{tabular}{lccc} 
Directions & Transverse $(\mathrm{cm})$ & Sagittal $(\mathrm{cm})$ & CT $(\mathrm{cm})$ \\
\hline 2. AP & 5.63 & 5.66 & 5.65 \\
\hline 5. LR & 4.60 & 4.56 & 4.30 \\
\hline 7. SI & 4.37 & 4.37 & 4.20 \\
\hline Volume $(\mathrm{ml})$ & 60.57 & 60.67 & 60.82
\end{tabular}

CT-computed tomography

Index numbers of directions were corresponding to that on Figure 1

caused by other factors. The prostate gland has some elasticity, and its shape is deformable. In TRUS image acquisitions, there is no doubt that probe insertion has the most significant effect on anatomical change. Several papers have reported anatomical and dosimetric changes due to probe insertion or rectal balloon insertion $[7,8,9]$. The rectum has a natural backward bend from the sigmoid colon to the anus. The curved portion is usually at the level of the prostate mid-gland or the apex; however, the probe insertion causes the rectum to straighten. Although the anus is tightly fixed by the pelvic floor muscles, the rectum is loosely fixed by the surrounding fat tissue. Therefore, the soft tissue of the prostate can be extended in the AP direction, as our study reveals.

However, prostate size in sagittal image acquisitions were not always larger than that in transverse image acquisitions. Our study suggests that patients with relatively smaller size of prostate apex in AP direction in sagittal image acquisitions compared to that in transverse image acquisitions, tended to have low $\mathrm{pD}_{90}$ and $\mathrm{pV}_{100}$ in post-implanted CT analysis. There was no clear explanation about this relationship in this study. One of possible explanations might be prostate rotation. Not only extension but also rotation could occurred during probe insertion, although no data was available in this study. We aim to conduct further study to quantify prostate rotation due to the TRUS probe. With regard to prostate volume, a report by Ali et al. also compared transverse and sagittal image acquisitions [10]. They reported that in a group of patients with a small prostate size, the prostate volume in the sagittal image acquisitions was larger than that in the transverse image acquisitions, although the opposite result was shown in the large $(>50 \mathrm{cc}$ ) prostate group. Since the patients in our study had relatively small prostates $(\leq 45 \mathrm{cc})$, our results were compatible with those of the small prostate group in the above-mentioned study.

There were several limitations that should be considered. Firstly, some slices were inevitably missed when performing axial or sagittal ultrasound acquisitions. Therefore, $2 \mathrm{~mm}$ or more gaps were presented in some patients, although most of images were acquired in $1 \mathrm{~mm}$ slice thickness. These gaps did not cause any contraction of prostate contours because the missing slices were automatically interpolated. Therefore, we believe that effects induced by these gaps would be minor. Secondly, because the initial plan of this study was only to compare the two types of image acquisitions, number of patients was set for this purpose only. Therefore, suggested relationships between DVH parameters and difference of the two types of image was not based on sufficient statistical power, and could not explained by available data. We are planning to investigate these issues in a further study.

\section{Conclusions}

Our study revealed that there were small but significant differences in the prostate contours between the transverse and the sagittal planning images. Furthermore, our study suggested that the differences between the two types of image acquisitions might correlated to dosimetric results on CT analysis. 
A

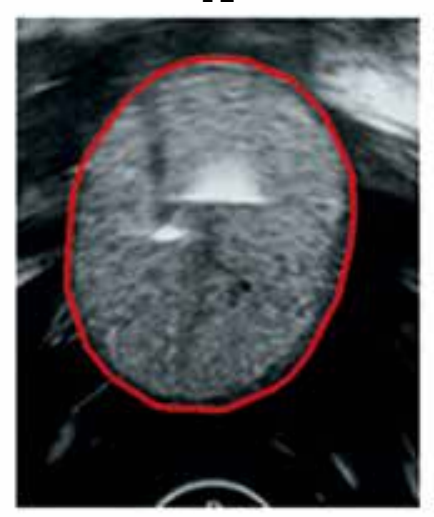

D

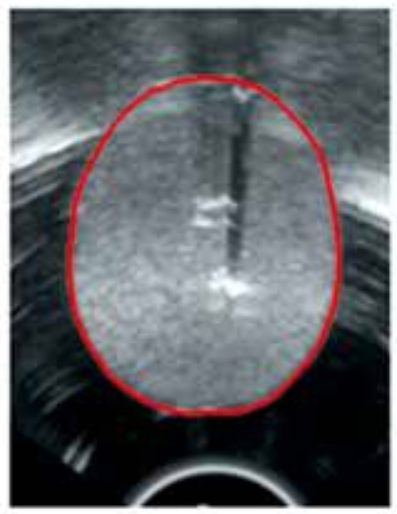

G

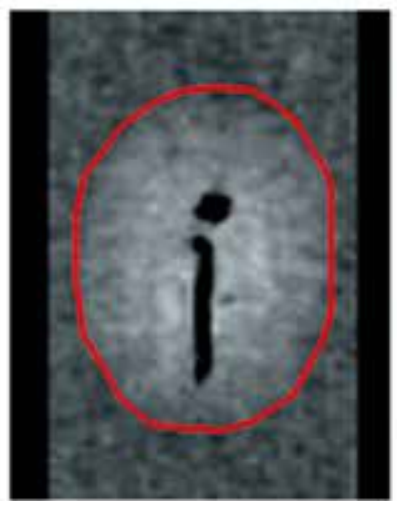

B

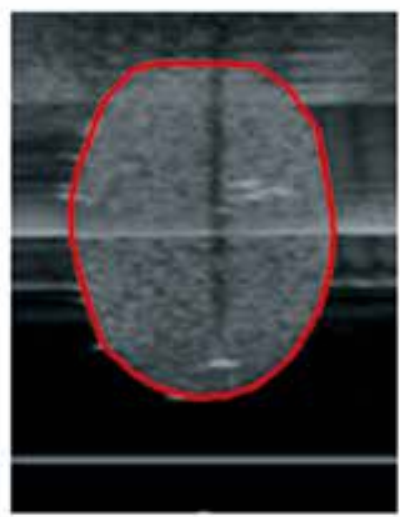

E

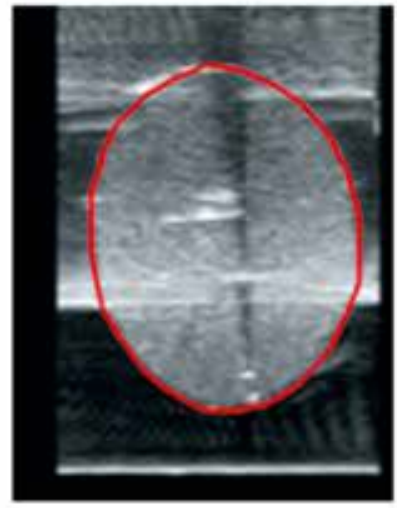

$\mathbf{H}$

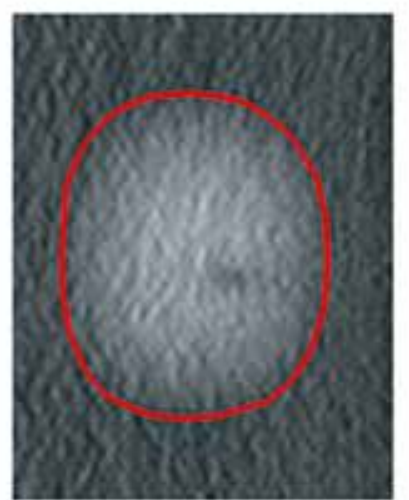

C

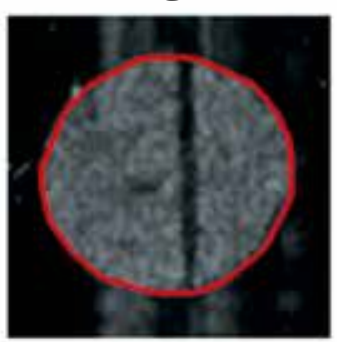

$\mathbf{F}$

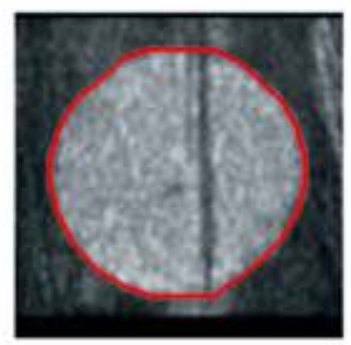

I

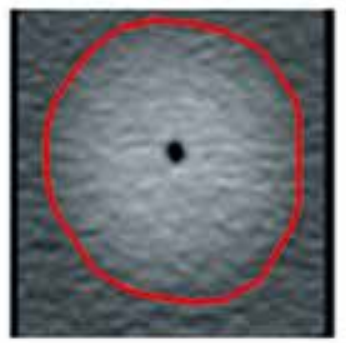

Fig. 5. Images of the phantom on the 2 types of transrectal ultrasound (TRUS) image acquisitions and on computed tomography (CT). There was no apparent differences among the transverse image acquisition (A-C), sagittal image acquisition (D-F), and CT images (G-I) on transverse (A, D, G), sagittal (B, E, H), and coronal (C, F, I) planes

\section{Disclosure}

Dr. Ishiyama reports other from Medicon Co., Ltd., other from Nihon Medi-Physics. Co., Ltd., during the conduct of the study.

\section{References}

1. Davis BJ, Horwitz EM, Lee WR et al. American Brachytherapy Society consensus guidelines for transrectal ultrasound-guided permanent prostate brachytherapy. Brachytherapy 2012; 11: 6-19.
2. Guinot JL, Ricós JV, Tortajada MI et al. Comparison of permanent (125)I seeds implants with two different techniques in 500 cases of prostate cancer. J Contemp Brachytherapy 2015; 7: $258-264$.

3. http://www.jastro.or.jp/customer/guideline/2016/10/ 977bff02b89c8ffbd591c47911d7a3e0c5ddf648.pdf.

4. Stock RG, Stone NN, Wesson MF et al. A modified technique allowing interactive ultrasound-guided three-dimensional transperineal prostate implantation. Int J Radiat Oncol Biol Phys 1995; 32: 219-225. 
5. Zauls AJ, Ashenafi MS, Onicescu G et al. Comparison of intraoperatively built custom linked seeds versus loose seed gun applicator technique using real-time intraoperative planning for permanent prostate brachytherapy. Int J Radiat Oncol Biol Phys 2011; 81: 1010-1016.

6. Ishiyama $\mathrm{H}$, Satoh $\mathrm{T}$, Kawakami $\mathrm{S}$ et al. A prospective quasi-randomized comparison of intraoperatively built custom-linked seeds versus loose seeds for prostate brachytherapy. Int J Radiat Oncol Biol Phys 2014; 90: 134-139.

7. Ishiyama $H$, Kitano $M$, Satoh $T$ et al. Difference in rectal dosimetry between pre-plan and post-implant analysis in transperineal interstitial brachytherapy for prostate cancer. Radiother Oncol 2006; 78: 194-198.

8. Seppenwoolde Y, Kolkman-Deurloo IK, Sipkema D et al. HDR prostate monotherapy: dosimetric effects of implant deformation due to posture change between TRUS- and CT-imaging. Radiother Oncol 2008; 86: 114-119.

9. Otón LF, Dolado MC, Núñez EJ et al. Effect of constipation on dosimetry after permanent seed brachytherapy for prostate cancer. J Contemp Brachytherapy 2015; 7: 247-251.

10. Ali I, Algan O, Thompson S et al. A comparative study of seed localization and dose calculation on pre- and post-implantation ultrasound and CT images for low-dose-rate prostate brachytherapy. Phys Med Biol 2009; 54: 5595-5611. 